\title{
Dental anxiety, psychiatry and dental treatment: How are they linked?
}

\author{
Salvatore Settineri ${ }^{1}$, Domenico Mallamace ${ }^{2}$, Maria Rosaria A. Muscatello ${ }^{2}$, Rocco Zoccali ${ }^{2}$, \\ Carmela Mento $^{2 *}$ \\ ${ }^{1}$ Department of Human and Social Sciences, University of Messina, Messina, Italy \\ ${ }^{2}$ Department of Neurosciences, University of Messina, Messina, Italy \\ Email: *
}

Received 24 October 2012; revised 25 November 2012; accepted 6 December 2012

\begin{abstract}
Background: The autonomy of dental anxiety may be compared with other psychopathologies, however, it is classified as indicative of a specific phobia. It is interesting to know how dental anxiety operates within a wider context. Material and Method: The group was made by 514 subjects, recruited from several dental surgeries. The entire process, consisted in selfcompilation of the Dental Anxiety Scale (DAS), and the Patient Health Questionnaire (PHQ). The software used for statistical calculations was the Statistical Package for Social Sciences (SPSS) version 16.0. The chi-square test was used for the distribution of the nominal variables. Results: The analysis of data revealed a significantly different distribution between high and low levels of dental anxiety and comorbidity in relation to only one diagnostic category, namely mood disorders. Dental fears and comorbidity were mostly in line with our expectations, except about fear of needles with eating disorders. Dental anxiety is to be considered as a dimension on a par with other psychopathologies. Conclusions: The study of comorbidity between mood disorders and dental anxiety, suggests that dental anxiety rather than belonging to the family of anxiety disorders would be better classified along with mood disorders. The findings regarding emotional inducers (fears) in relation to the distribution of psychopathology are worth noting. The above observations reaffirm the need to form links between dentistry and psychiatry.
\end{abstract}

Keywords: Dental Anxiety; Fears; Psychiatric Comorbidity

\section{INTRODUCTION}

Compliance with dental treatment is affected by emotions. Emotions could thus become pathological when

"Corresponding author. they play a role in clinical situations where there is a lack of balance between real and perceived danger, such as in dental anxiety.

Dental anxiety is defined as the response to a stressful stimulus that is specific to a dental context. This definition, which is shared by the literature, contains two coexisting aspects [1]. The first is linked to anxiety as a feeling and the second is linked to its recognition by reference to its origin. In this sense dental anxiety is translated, on the one hand as an affective expression of a normal anxious state, and on the other hand as a pure and specific psychopathological condition.

Some studies have shown that dental anxiety depends on self-consciousness of treatment [2,3]. In general selfconsciousness is defined as the perception of oneself, and more specifically as the tendency to think about and evaluate aspects of oneself that are subjected to stressful events (for example dental stimuli). It is for this is the reason that oral procedures are perceived as being so stressful that they can cause acute symptoms of anxiety, such as excessive apprehension, irritability, tension due to anticipated harm and avoidance of treatment $[4,5]$. The diagnostic autonomy of dental anxiety may be compared with other psychopathologies, however this autonomy is not recognized in the chapter on anxiety disorders in the Diagnostic and Statistical Manual of Mental Disorders DSM [6]. In contrast, criteria that express excessive or unreasonable fear or the avoidance of exposure to stimuli (e.g. blood, injections or other medical stimuli) are classified as being of a specific phobia by the DSM- IV. It is clearly reductionist to apply the classic concept of phobia to a phenomenon that leads to anxiety but which does not necessarily present with the characteristics of a classic phobia and this is why dental anxiety should be considered a distinct condition [7-9]. Greater understanding could be obtained if attention were to be shifted away from the context in which dental anxiety is generated and focused instead on the overall psychopathological profile of the subject. 
Studies on comorbidity of medical conditions as well as psychiatric disorders have produced some interesting findings that aid understanding of what seem to be distinct clinical conditions [10-12]. The reason for comorbidity lies in the fact that emotions are in any case represented by the body and it is the degree to which the subject is conscious of these emotions that makes them manifest $[13,14]$.

The aim of this study is to ascertain whether or not basic diagnostic categories are linked to dental anxiety. The study has been designed to consider anxiety arising from representational stimuli is considered separately from the anxiety originating from dentist-patient relations and also phobic items are also dealt with apart.

\section{METHODS}

\subsection{Subjects and Procedure}

544 subjects took part in this study but 30 were excluded because they failed to respond to important items on the questionnaire. The group, thus comprising 514 subjects, was recruited from several dental surgeries. 253 subjects were male and 261 female and they were aged between 16 and 70 years old (average $=35.70$; S.D. $=14.21$ ). After obtaining their consent, the subjects were asked to take part in the study during the time they spent in the waiting room. Prior consent was also obtained from the dentist.

Potential subjects were excluded from the study if they were: 1) under 16 years or over $70 ; 2$ ) presented signs of neurological disease; 3 ) were using psychoactive drugs.

\subsection{Questionnaire}

The entire process, with the exception of the introduction and briefing, consisted in self-compilation of the Dental Anxiety Scale (DAS) and the Patient Health Questionnaire (PHQ).

1) Anxious behavior by subjects in the dental treatment room was assessed using the Dental Anxiety Scale (DAS) containing 19 sub-scales $[15,16]$. This scale is divided into two parts.

The first part (DAS 1) is made up of 6 sub-scales, of which the first 5 explore the traits of dental anxiety of the patient and the replies are given using a score from 0 to 4 , with the total score for this first part ranging between 0 and 20. The total score was considered to indicate a low anxiety level if $<14$ and a high level where $>15$ [16].

Sub-scale 6 of DAS 1 looks at dental anxiety induced by specific dental stimuli using 6 items, (injection needles (6a), drill noise (6b), pain of treatment (6c), the smell of teeth being drilled (6d), a feeling of suffocation/gagging/lack of air (6e), the reclined position of the dentist chair (6f). Each answer was scored from 1 to 7.

The second part (DAS 2), containing 13 sub-scales, explores dental anxiety relating to dentist-patient relations and replies are assigned a score on a descending scale from 2 to 0 , with a cumulative score range of between 0 and 26. A judgement of the professionalism or respect for the dentist is implicit in all the items. Anxiety level was classified either as low (total score $<12$ ) or high (total score $>13$ ).

2) The identification of specific mental disorders (somatoform, depressive, dysthymic, anxiety, panic, eating and substance abuse) was evaluated using the Patient Health Questionnaire (PHQ) comprising 10 sub-scales $[17,18]$. The choice of this questionnaire was governed by its ability, documented in the literature, to examine the main psychiatric pathologies [19].

In this study the following categories were utilized: mood disorders (18 items including depression and dysthymia) somatoform disorders (13 items including somatization, anxiety somatized under the form of analgesia in various parts of the body) anxiety disorders (12 items including panic attacks and other anxiety related syndromes) eating disorders ( 8 items including bulimia nervosa and binge-disorder) and disorders linked to substance abuse (3 items including the frequent use of alcohol). The replies were given a score of 0 to indicate the absence or 1 the presence of the disorder on the basis of diagnostic criteria.

\subsection{Statistical Analysis}

The software used for statistical calculations was the Statistical Package for Social Sciences (SPSS) version 16.0. The following numeric variables were explored separately: DAS 1; DAS 2 and specific fears (sub-scales 6 , six items 6a-f) of DAS 1 .

The values for DAS 1 and DAS 2 were classified as either medium-high or medium-low, according to their intensity. Assignment followed Corah where for DAS 1, a total score of $<14$ was considered a medium-low anxiety level and a score of $>15$ medium-high. Similarly for DAS 2, a score of $<12$ was considered a medium-low anxiety level and $>13$ medium-high. The total score of the specific fears investigated (DAS 1 -sub-scale 6) was also divided into two categories: a medium-low score (1 - 3) and medium-high (4 - 7).

The variables in the PHQ were considered to assess whether or not there was comorbidity of dental anxiety with other psychiatric conditions. The chi-square test was used for the distribution of these variables.

\section{RESULTS}

\subsection{Dental Anxiety Arising from Representational Factors and Comorbidity}

The following results were obtained when considering 
the group overall $(\mathrm{n}=514)$.

As regards dental anxiety explored by DAS 1, 28 subjects $(5.4 \%)$ were found to have medium-high anxiety. The comorbidity of this dimension is presented in Table 1. The analysis of data revealed a significantly different distribution between high and low levels of this form of dental anxiety and comorbidity in relation to only one diagnostic category, namely mood disorders.

The DAS 1 and DAS 2 were compared using standardised Z-scores. The comparison between the Z-scores of DAS 1 (average $=1.15-$ NS) and DAS 2 (average $=$ 1.361 - NS) showed no significant differences for either. DAS 1 and DAS 2 were positively correlated $(r=0.192$; $\mathrm{p}=0.01)$.

No significant differences emerged in relation to the subjects' sex or age.

\subsection{Anxiety Dentist-Patient Relations and Comorbidity}

The following results were obtained considering the group overall $(\mathrm{n}=514)$ : 116 subjects $(22.5 \%)$ were found to have medium-high DAS 2 anxiety levels. Table 2 shows the comorbidity of this dimension. The analysis of data showed a significantly different distribution between high and low levels of this form of anxiety with only one diagnostic category, mood disorders.

No significant differences emerged in relation to the subjects' sex or age.

\section{DISCUSSION}

The associations and the differences observed between dental anxiety and other associated psychiatric disorders have led us to propose the following hypotheses:

Dental anxiety is to be considered as a dimension on a par with other psychopathologies and therefore an aspect of mental functioning that goes from the normal to the pathological, once it exceeds a certain threshold. Comorbidity is important when the presence of other psychiatric disorders differs from the one expected in terms of statistical distribuction [20-23].

The correlation between dental anxiety arising from interpersonal relations (as explored by DAS 2) and the anxiety deriving from representational factors (i.e. linked to the images evoked, as explored by DAS 1) also found in our study leads us speculate on the role played by consciousness in both of these, as it seems to confirm published work that highlights the role played by selfconsciousness [1-3].

The study of comorbidity between mood disorders and dental anxiety, in both its forms, suggests that dental anxiety rather than belonging to the family of anxiety disorders would be better classified along with mood disorders [13]. We would not seem to be the only ones to make this claim given the emphasis of literature on the continuum of mood disorders and anxiety disorders [24-26].

Our study carried out on a significant sample population found that the relevance of results arises from the context in wich the study was carried out, i.e. the waiting room of a dental surgery.

The emotional response found was lower than we expossible therefore that dental anxiety was denied or discharged at the time of treatment, complicating the pat tern.

Table 1. Chi-square test: DAS 1 comorbidity with nosographic categories.

\begin{tabular}{|c|c|c|c|c|}
\hline Nosographic & Anxiouses & \multicolumn{3}{|c|}{ Non-anxiouses } \\
\hline Categories & $\mathrm{n}=28(5.4 \%)$ & $\mathrm{n}=486(94.5 \%)$ & $\chi^{2}(\mathrm{df}=1)$ & Sig. \\
\hline \multicolumn{5}{|l|}{ Mood disorders } \\
\hline Absent & $22(4.28 \%)$ & $441(85.79 \%)$ & 4.887 & $\mathrm{p}=0.022$ \\
\hline Present & $6(1.16 \%)$ & $45(8.75 \%)$ & & \\
\hline \multicolumn{5}{|c|}{ Somatoform disorders } \\
\hline Absent & $21(4.08 \%)$ & $425(82.68 \%)$ & 3.574 & $\mathrm{p}=0.297$ \\
\hline Present & $7(1.36 \%)$ & $61(11.86 \%)$ & & \\
\hline \multicolumn{5}{|l|}{ Anxiety disorders } \\
\hline Absent & $24(4.66 \%)$ & $433(84.24 \%)$ & 0.307 & $\mathrm{p}=0.772$ \\
\hline Present & $4(0.77 \%)$ & $53(10.31 \%)$ & & \\
\hline \multicolumn{5}{|l|}{ Eating disorders } \\
\hline Absent & $25(4.86 \%)$ & $443(86.18 \%)$ & 0.113 & $\mathrm{p}=0.550$ \\
\hline Present & $3(0.58 \%)$ & $43(8.36 \%)$ & & \\
\hline \multicolumn{5}{|c|}{ Substance abuse disorders } \\
\hline Absent & $28(5.44 \%)$ & $446(86.77 \%)$ & 2.499 & $\mathrm{p}=0.233$ \\
\hline Present & 0 & $40(7.78 \%)$ & & \\
\hline
\end{tabular}


Table 2. Chi-square test: DAS 2 comorbidity with nosographic categories.

\begin{tabular}{|c|c|c|c|c|}
\hline Nosographic & Anxiouses & \multicolumn{3}{|c|}{ Non-anxiouses } \\
\hline Categories & $\mathrm{n}=116(22.5 \%)$ & $\mathrm{n}=398(77.4 \%)$ & $\chi^{2}(\mathrm{df}=1)$ & Sig. \\
\hline \multicolumn{5}{|l|}{ Mood disorders } \\
\hline Absent & $98(19.06 \%)$ & $365(7.10 \%)$ & 5.247 & $\mathrm{p}=0.022$ \\
\hline Present & $18(3.50 \%)$ & $33(6.42 \%)$ & & \\
\hline $\begin{array}{l}\text { Somatoform disorders } \\
\text { Absent } \\
\text { Present }\end{array}$ & $\begin{array}{c}104(20.23 \%) \\
12(2.33 \%)\end{array}$ & $\begin{array}{c}342(66.35 \%) \\
56(10.89 \%)\end{array}$ & 1.086 & $\mathrm{p}=0.297$ \\
\hline $\begin{array}{l}\text { Anxiety disorders } \\
\text { Absent } \\
\text { Present }\end{array}$ & $\begin{array}{c}104(20.23 \%) \\
12(2.33 \%)\end{array}$ & $\begin{array}{c}353(68.67 \%) \\
45(8.75 \%)\end{array}$ & 0.084 & $\mathrm{p}=0.772$ \\
\hline \multicolumn{5}{|l|}{ Eating disorders } \\
\hline $\begin{array}{l}\text { Absent } \\
\text { Present }\end{array}$ & $\begin{array}{c}104(20.23 \%) \\
12(2.33 \%)\end{array}$ & $\begin{array}{c}364(70.81 \%) \\
34(6.61 \%)\end{array}$ & 0.358 & $\mathrm{p}=0.550$ \\
\hline $\begin{array}{c}\text { Substance abuse disorders } \\
\text { Absent } \\
\text { Present }\end{array}$ & $\begin{array}{c}110(21.40 \%) \\
6(1.16 \%)\end{array}$ & $\begin{array}{c}364(70.81 \%) \\
34(6.61 \%)\end{array}$ & 1.422 & $\mathrm{p}=0.233$ \\
\hline
\end{tabular}

The psysician should differentiate what is linked to a state (dental anxiety) from the situation arising from a psychiatric disorder. The incidence of each diagnostic category in relation to mood disorders was lower than that generally found for the general population. This might be related to the diagnostic method employed one based empirically on standardised scales.

The use of the DAS allows the specific measurement of anxiety provoked by dental stimuli and this is demonstrated by its widespread use in the literature $[21,22,26]$.

The above observations reaffirm the need to forge links between dentistry and psychiatry [27-29]. The dentist should be able to make an accurate anamnesis and to recognize and deal with dental patients who may suffer any psychiatric disorders in order to enhance patient compliance and treatment.

\section{REFERENCES}

[1] Economou, G.C. and Honours, B. (2003) Dental anxiety and personality: Investigating the relationship between dental anxiety and self-consciousness. Journal of Dental Education, 267, 970-980.

[2] Samorodnitzky, G.R. and Levin, L. (2005) Self-assessed dental status, oral behavior, DMF, and dental anxiety. Journal of Dental Education, 69, 1385-1389.

[3] Kloostra, P.W., Eber, R.M. and Inglehart, M.R. (2007) Anxiety, stress, depression, and patient's responses to periodontal treatment: Periodontists' knowledge and pro- fessional behavior. Journal of Periodontology, 78, 64-71. doi:10.1902/jop.2007.060069

[4] Locker, D. (2003) Psychological consequences of dental fear and anxiety. Community Dentistry and Oral Epidemiology, 31, 144-151. doi:10.1034/j.1600-0528.2003.00028.X

[5] Anttila, S., Knuuttila, M., Ylöstalo, P. and Joukamaa, M. (2006) Symptoms of depression and anxiety in relation to dental health behavior and self-perceived dental treatment need. European Journal of Oral Sciences, 114, 109-114. doi:10.1111/j.1600-0722.2006.00334.x

[6] American Psychiatric Association (1994) Diagnostic and statistical manual of mental disorders. 4th Edition, DSM, Washington DC.

[7] Majstorovic, M. and Veerkamp, J.S. (2004) Relationship between needle phobia and dental anxiety. Journal of Dentistry for Children, 71, 201-205.

[8] TarjaKaakko, D.D.S., Susan, E., Coldwell, Ph.D., Tracy Getz, M.S., Peter Milgrom, D.D.S., Peter, P., Roy-Byrne, M.D., Douglas, S. and Ramsay, D.M.D. (2000) Psychiatric diagnoses among self-referred dental injection phobics. Journal of Anxiety Disorder, 10, 299-312.

[9] De Jong, A., Bongaart, G., Vermeule, I., Visser, K., De Vos, P. and Makkes, P. (1998) Blood-injection-injury phobia and dental phobia. Behaviour Research and Therapy, 36, 971-982. doi:10.1016/S0005-7967(98)00064-3

[10] Xue, Z.X., Cui, T., Hou, R., Ju, Y. and Lu, B. (2004) Prevalence of dental anxiety in 144 patients with cardiovascular disease before tooth extraction. Zhonghua Liu Xing Bing Xue ZaZhi, 25, 531-533. 
[11] Mathew, R.J., et al. (1979) Xerostomia and sialorrhea in depression. American Journal of Psychiatry, 136, 14761477.

[12] Simmons, M.S., Grayden, S.K. and Mitchell, J.E. (1986) The need for psychiatric-dental liaison in the treatment of bulimia. American Journal of Psychiatry, 143, 783-784.

[13] Marques-Vidal, P. and Milagre, V. (2006) Are oral health status and care associated with anxiety and depression? A study of Portuguese health science students. Journal of Public Health Dentistry, 66, 64-66. doi:10.1111/j.1752-7325.2006.tb02553.x

[14] Majstorovic, M., Skrinjarìc, T., Szirovicza, L., Glavina, D. and Veerkamp, J.S. (2007) Dental anxiety in relation to emotional and behavioral problems in Croatian adolescents. Collegium Antropologicum, 31, 573-578.

[15] Corah, N.L. (1969) Development of a dental anxiety scale. Journal of Dental Research, 48, 596. doi:10.1177/00220345690480041801

[16] Corah, N.L. et al. (1978) Assessment of a dental anxiety scale. Journal of American Dental Association, 97, 816819.

[17] Spitzer, R.L. and Williams, J.B.W. (1994) Utility of a new procedure for diagnosing mental disorders in primary care: The PRIME-MD 1000 study. The Journal of the American Medical Association, 272, 1749. doi:10.1001/jama.1994.03520220043029

[18] Spitzer, R.L. and Kroenke, K. (1999) Validation and utility of a self-report version of PRIME-MD. The Journal of the American Medical Association, 282, 1737. doi:10.1001/jama.282.18.1737

[19] Becker, S., Al Zaid, K. and Al Faris, E. (2002) Screening for somatization and depression in Saudi Arabia: A validation study of the PHQ in primary care. International Journal of Psychiatry in Medicine, 32, 271-283. doi:10.2190/XTDD-8L18-P9E0-JYRV

[20] Moore, R., Brodsgaard, I. and Rosenberg, N. (2004) The contribution of embarrassment to phobic dental anxiety:
A qualitative research study. BMC Psychiatry, 4, 10.

[21] Maggirias, J. and Locker, D. (2002) Five-year incidence of dental anxiety in an adult population. Community Dental Health, 19, 173-179.

[22] Locker, D., Thomson, W.M. and Poulton, R. (2001) Psychological disorder, conditioning experiences, and the onset of dental anxiety in early adulthood. Journal Dental Research, 80, 1588-1592. doi:10.1177/00220345010800062201

[23] Locker, D., Thomson, W.N. and Poulton, R. (2001) Onset and patterns of change in dental anxiety in adolescence and early adulthood: A birth cohort study. Community Dental Health, 18, 99-104.

[24] Yerevanian, B.I., Koek, R.J. and Swarnalatha, R. (2001) Anxiety disorders comorbidity in mood disorder subgroups: Data from a mood disorders clinic. Journal of Affective Disorder, 67, 167-173. doi:10.1016/S0165-0327(01)00448-7

[25] Zimmerman, M. and Iwona Chelminski, M.D. (2003) Generalized anxiety disorder in patients with major depresssion: Is DSM-IV's hierarchy corret? American Journal of Psychiatry, 160, 504-512. doi:10.1176/appi.ajp.160.3.504

[26] Kaspers, S. (2001) Depression and anxiety-separate or continuum? World Journal Biological Psychiatry, 162, 162-163. doi:10.3109/15622970109026804

[27] Jören, P., Jackowski, J., Gängler, P., Sartory, G. and Thom, A. (2000) Fear reduction in patients with dental treatment phobia. British Journal of Oral and Maxillofacial Surgery, 38, 612-616. doi:10.1054/bjom.2000.0531

[28] De Jongh, A. (2003) Clinical characteristics of somatization in dental practice. British Dental Journal, 9, 151154.

[29] Settineri, S., Mento, C., Gugliotta, S.C., Saitta, A., Terranova, A., Trimarchi, G. and Mallamace, D. (2010) Selfreported halitosis and emotional state: Impact on oral conditions and treatments. Health and Quality of Life Outcomes, 8, 34. doi:10.1054/bjom.2000.0531 